\title{
Delirium in Intensive Care
}

\author{
Lone Musaeus Poulsen $^{1}(1) \cdot$ Stine Estrup ${ }^{2} \cdot$ Camilla Bekker Mortensen $^{1} \cdot$ Nina Christine Andersen-Ranberg ${ }^{1}$
}

Accepted: 6 July 2021 / Published online: 3 September 2021

(c) The Author(s), under exclusive licence to Springer Science+Business Media, LLC, part of Springer Nature 2021

\begin{abstract}
Purpose of Review Delirium in the intensive care unit (ICU) has become increasingly acknowledged as a significant problem for critically ill patients affecting both the actual course of illness as well as outcomes. In this review, we focus on the current evidence and the gaps in knowledge.

Recent Findings This review highlights several areas in which the evidence is weak and further research is needed in both pharmacological and non-pharmacological treatment. A better understanding of subtypes and their different response to therapy is needed and further studies in aetiology are warranted. Larger studies are needed to explore risk factors for developing delirium and for examining long-term consequences. Finally, a stronger focus on experienced delirium and considering the perspectives of both patients and their families is encouraged.

Summary With the growing number of studies and a better framework for research leading to stronger evidence, the outcomes for patients suffering from delirium will most definitely improve in the years to come.
\end{abstract}

Keywords Delirium · Intensive care · CAM-ICU · Risk factors · Non-pharmacological $\cdot$ Pharmacological · Core outcome

\section{Introduction}

Delirium is an acute brain dysfunction often associated with critical illness. The term delirium derives from the Latin "de lira" meaning out of furrow or out of track and referring to the confusion often characterizing the condition. Since the beginning of the twenty-first century publications regarding intensive care unit (ICU)-acquired delirium have been increasing exponentially acknowledging the condition as a significant burden for the critically ill patients. Delirium has

This article is part of the Topical Collection on Critical Care Anesthesia

Lone Musaeus Poulsen

lmp@regionsjaelland.dk

Stine Estrup

sed@regionsjaelland.dk

Camilla Bekker Mortensen

cbem@regionsjaelland.dk

Nina Christine Andersen-Ranberg

ncan@ regionsjaelland.dk

1 Department of Anaesthesiology, Zealand University Hospital, Lykkebækvej 1, 4600 Koege, Denmark

2 Department of Intensive Care Medicine, Rigshospitalet, Blegdamsvej, 2100 Copenhagen, Denmark been documented as common in the ICU population and associated with numerous and deleterious outcomes. This review will provide an overview of the current evidence, discuss some of the gaps in knowledge, and suggest future focus for research in ICU-acquired delirium.

\section{Definition of Delirium}

Delirium is and continues to be described with inconsistent terminology such as ICU syndrome, acute brain dysfunction, and septic encephalopathy depending on geographic location and medical specialty. The term delirium should therefore be acknowledged as an umbrella noun adopted to overcome all these terms [1]. Despite the inconsistency in terminology, there is a general consensus of the delirium definition which is based on the Diagnostic and Statistical Manual of the American Psychiatric Association (DSM-5) [2]. The DSM-5 diagnostic criteria for delirium include the following:

- Disturbance of consciousness (i.e. reduced clarity of awareness of the environment)

- Change of cognition (e.g. memory deficit, disorientation, language disturbance, perceptual disturbance) 
- The disturbance develops over a short period of time (usually hours to days) and tends to fluctuate during the course of the day

- Evidence from the history, physical examination, or laboratory findings that indicates the disturbance is caused by a direct physiologic consequence of a general medical condition, an intoxicating substance, medication use, or more than one cause [2].

Attention deficits and cognitive changes cannot be explained by pre-existing neurocognitive disturbances (e.g. dementia) or a significantly decreased level of consciousness (e.g. sedation or coma). Delirium should be differentiated from dementia by the course of disease development. While delirium is characterised as an acute and fluctuating change in cognition, dementia is characterized as a condition of generalized cognitive deficits, impaired memory and intellectual abilities evolved over a considerably longer period of time (months, years) compared to delirium [3]. The diagnosis of delirium identifies the disturbances of central nervous system (CNS) function, yet does not recognise their aetiology; therefore, the cause of dysfunction should be sought immediately.

The clinical presentation of delirium varies tremendously. Delirium is typically divided into 3 clinical subclasses: hyperactive, hypoactive and mixed motor subtype based on the predominant psychomotor activity. The hypoactive patient has slowed mentation, lethargy, and decreased movements, while the hyperactive patient has an increased number of spontaneous movements that are purposeless, uncontrollable, and inefficient. A delirious patient may fluctuate between a hypoactive and hyperactive state and the delirium is then termed mixed form [4]. While hyperactive motor subtype is the easiest to detect in clinical practice it is the least prevalent. Hypoactive motor subtype is the most common motor subtype in the ICU but is easily overlooked due to its silent clinical picture [5].

\section{How to Measure Delirium}

The Confusion Assessment Method for the Intensive Care Unit (CAM-ICU) and the Intensive Care Delirium Screening Checklist (ICDSC) are the most frequently used assessment tools for delirium [6-8]. Delirium screening using the CAMICU or the ICDSC is quick, lasting only $2-5 \mathrm{~min}[9,10]$. Both tools have very good psychometric properties and moderate to high interrater reliability [8]. Sensitivity and specificity of the tools have been evaluated in the Pain Agitation and Delirium (PAD) guideline from 2013 and a subsequent systematic review $[8,11]$. CAM-ICU has been tested in more than 4000 adult ICU patients and has been translated and validated into 27 languages. Reported sensitivity for CAM-ICU ranged from
47 to $100 \%$ while specificity ranged from 81 to $100 \%$. The ICDSC has been tested in more than 2500 adult ICU patients and translated and validated in 6 languages. The sensitivity has been reported to range from 64 to $99 \%$ and specificity between 61 and $88 \%$ [8]. Multiple studies in the ICU setting have found that without validated screening tools, bedside nurses and physicians fail to recognize delirium [12-14].

Clinical practice guidelines have issued strong recommendations for the use of routine delirium assessment with a validated assessment tool in the ICU $[11,15 \bullet \bullet]$. Although it is difficult to find effect of routine delirium assessment on outcomes of delirium, the potential benefits of delirium screening outweigh the harms. The most important benefit of delirium monitoring is early recognition. Early detection may lead to prompt identification and correction (when possible) of aetiology, assurance of patients experiencing distressing symptoms, treatment (pharmacologic or nonpharmacologic), and treatment effectiveness assessments. Furthermore, to improve research in delirium, evaluate treatment effects on delirium and improve communication between clinicians, nurses, relatives, patients and researchers, a systematic and reliable screening of delirium is necessary $[15 \bullet \bullet, 16]$.

\section{Delirium Incidence and Prevalence}

Delirium is frequently encountered in the ICU. A recent systematic review based in 48 studies found a pooled prevalence of delirium to be $31 \%$ (95\% CI 24-41), while a pooled incidence was reported to $22 \%$ (95\% CI 16-31). Many patients are exhibiting symptoms of delirium at admission to an ICU, which is why delirium prevalence is the most accurate measure of the delirium burden in the ICU [5].

As motoric subtypes of delirium have been shown to have implication for patient prognosis and detection of delirium, classification of delirium into motoric subtypes is becoming more prevalent $[4,17]$. The prevalence of hypoactive delirium is $17 \%$ (95\% CI 13-22), followed by mixed motor subtype $10 \%$ (95\% CI 6-16), and lastly hyperactive motor $4 \%$ (95\% CI 3-6) [5]. This gives reason to speculate whether the different motor subtypes actually demonstrate different diseases which should be managed differently and that the lack of acknowledgement of these differences might be the reason for the inability to find an effective treatment for delirium.

\section{Pathophysiology}

In order to better understand the acute dysfunction of the delirious brain at a structural level, various neuroimaging technics have been employed. It has provided evidence of a widespread brain dysfunction leading to cell death 
and atrophy of the central nervous tissue. Cerebral blood flow seems to be affected during delirium and if this disturbance is maintained over a period of time it may trigger apoptosis of neural tissue [18]. CT scans support the link between delirium and structural changes in the brain. Images of the delirious brain have identified global lesions in the white matter as well as lesions in the basal ganglia [19].

On the neurotransmitter level, several substances seem to be involved. Imbalances in synthesis, release, and degradation of acetylcholine, gamma-aminobutyric acid (GABA), glutamate, serotonin, norepinephrine, and dopamine have been suggested to play important roles in the development of delirium [20]. Disturbed neurotransmission has the potential to cause changes in functional connectivity of the neurons and could be the trigger of atrophy and brain damage [4, 21] The most studied neurotransmitter pathway is the cholinergic. Acetylcholine is regulated by dopamine receptors and dopamine excess is depressing acetylcholine production [22]. Depressed acetylcholine production is induced by medications often used for the critically ill patients thus increasing the risk of delirium [23]. Precursors of neurotransmitters are also affected in delirium. The large neutral amino acids phenylalanine, tryptophan, tyrosine, and valine serve as precursors for serotonin, melatonin, dopamine, and norepinephrine. The relationship between these precursors is competitive meaning that an increase in the level of one might decrease the levels of the other impacting the imbalance between neurotransmitters [24].

When it comes to aetiology, two hypotheses describe precipitating factors of delirium. One hypothesis deals with factors that result in direct brain injury and another try to explain some of the more unclear mechanisms caused by the aberrant stress response [25].

The factors resulting in direct brain insults include processes that compromise brain function by energy deprivation, metabolic abnormalities, trauma, haemorrhage, or direct changes in neurotransmitter levels by drugs. This theory, though, does not account for the fact that subtler insults combined with pre-existing illness or age-related changes might also cause harm to the brain [25].

The hypothesis of the aberrant stress response suggests that the brain is subject to improper reactions or overstimulation due to inflammation or stressors. These inflammatory and stress responses occur particularly in vulnerable individuals and have been linked to delirium. There are two ways of explaining this phenomenon either the target tissue in the brain exhibits an exaggerated response to stimulation or vice versa an increased level of stimulation causes abnormal stress to the brain. The first one is called exaggerated cytokine-induced Sickness Behaviour while the second is known as limbic hypothalamic-pituitary-adrenal (LHPA) axis dysfunction [25]. The hypothesis is based on observations of patients with chronic cognitive impairment who develops delirium in response to mild precipitating factors.

Sickness Behaviour is the normal physiological adaptive response to systemic inflammation and is characterized by fatigue, reduced activity and reduced appetite. The purpose of this behaviour is conservation of energy thus minimizing exposure to other stressors [26]. Mediation of the response is to some extent performed by pro-inflammatory cytokines and prostaglandins [27] interacting directly with neurons and brain endothelium. Blood-brain barrier changes with progressing neurodegeneration in, e.g. ageing, diabetes, and Alzheimer's disease may increase the impact of inflammatory mediators causing increased inflammation in the CNS [28]. This might explain why an inflammatory event causes a normal physiological reaction in some individuals but triggers delirium in others.

LHPA is caused by failed down-regulation of the stress response following an acute event. This dysfunction results in sustained high levels of cortisol. This dysfunction caused by glucocorticoids affects cognition and attention and sustained levels may precipitate delirium. In several neurodegenerative diseases and in ageing an association between delirium and sustained high levels of cortisol has been identified [29]. Research continues to elaborate on the pathophysiology of delirium and further clarification on these theories are warranted.

\section{Risk Factors}

Different taxonomies are used to classify delirium risk factors. The most common is dividing risk factors into predisposing and precipitating factors and traditionally considering most precipitation factors as reversible. However, not all precipitating factors are reversible as not all predisposing factors are non-reversible. In order to shed more light on the factors amenable to intervention, a classification of risk factors into baseline characteristics, features of acute illness, and iatrogenic factors might prove more feasible [24].

Additionally, the evidence for most risk factors for delirium in hospitalized patients is obtained in non-ICU cohorts. It is generally accepted, though, that the risk factors for delirium are mutual in non-ICU and ICU settings.

Among the baseline characteristics for delirium in ICU, there is strongest evidence of association between the following risk factors and delirium: higher age, dementia, prior coma and pre-ICU emergency surgery or trauma. There is moderate evidence for hypertension, admission caused by neurological conditions and use of psychoactive medications (e.g. antipsychotics, anticonvulsants) as risk factors for delirium. The evidence is very weak or non-existing for gender and nicotine use [15••]. 
Higher disease severity measured by the Acute Physiology And Chronic Health Evaluation (APACHE) score and higher American Society of Anesthesiologists (ASA) classification of physical health are strongly associated with delirium as well [15••].

Finally, the iatrogenic risk factors with the strongest evidence for association with delirium are some medications and blood transfusion [15••].

Among risk factors with more moderate evidence are sedatives and analgesics, which are widely used in the ICU setting. Benzodiazepines, in particular lorazepam, is an independent risk factor for delirium and has been shown to increase odds of delirium by $20 \%$ for every milligram administered [30]. In contrast, the association between opioids and delirium is more unclear. In some studies, there is a strong association with delirium, but it is not a consistent finding throughout the literature [24]. An explanation could be the different indications for the use of opioids. When used as a sedative there seems to be a strong association between opioids and delirium but not if the indication for opioid is pain [31]. The evidence is very weak for mechanical ventilation and renal replacement therapy $[15 \bullet \bullet]$.

\section{Prediction}

The effect of reducing reversible risk factors on delirium burden and outcomes are unknown. In the end, it might be a patient's overall risk of delirium that determines the sensitivity to preventive measures or interventions [32]. Prediction models for delirium incorporate clinical risk factors (age, comorbidity, disease severity) and calculates individual risk scores for patients [33]. Prediction models may thereby assist the clinician in identifying patients at high risk of developing delirium and enable them to increase surveillance for early recognition of delirium and to introduce or intensify preventive measures [34, 35].

In order to prove feasible in clinical practice, a predictive model should be reproducible and generalizable. To date, only the PREdiction of DELIRium in ICu patients (PREDELIRIC) model has undergone both internal and external validation [36]. This model includes risk factors for delirium at ICU admission and within the first $24 \mathrm{~h}$ of ICU stay and has showed moderate power to predict delirium in ICU patients $[37,38]$. The need for predictors during the first $24 \mathrm{~h}$ of ICU admission might delay the identification of vulnerable patients at high risk for delirium. This has led many clinicians to find the Early PREdiction model for DELIRium in ICu patients (E-PRE-DELIRIC) model more feasible [39].

In general, many studies on delirium risk factors are challenged by confounding and future studies should make efforts to account for this $[15 \bullet \bullet]$.

\section{Pharmacological Treatment of Delirium}

Various pharmacologic agents including antipsychotics, statins, steroids, melatonin, opioids, benzodiazepines, and dexmedetomidine are used against delirium in the clinical setting [40, 41]. A recent international, cohort study investigating pharmacological interventions for delirium, found on the basis of data from 99 ICUs and 1200 ICU patients, that haloperidol was the most frequently used agent to treat delirium followed by benzodiazepines and dexmedetomidine [42]. An updated Cochrane review published in 2019 on pharmacological interventions for the treatment of delirium in critically ill adults found no pharmacological intervention to have an effect on delirium duration, mortality, duration of mechanical ventilation and other patient important outcomes. The most promising agent was the alpha2 agonist dexmedetomidine where analysis of only one small study showed that the agent may have some role in shortening duration of delirium. Most importantly, the review identified 10 ongoing studies, of which seven have a large target enrolment number (100 to 1000 participants); these RCTs may alter the conclusions of the review [43].

Research in delirium treatment is challenged by the fact that very few studies are actual treatment studies, meaning that a study investigating treatment effect needs to only include delirious patients, defined by a validated screening tool, to actually evaluate treatment effect. Many studies that are included in meta-analysis are in fact studies that include patients with high risk of delirium, e.g. mechanically ventilated patients and not per se patients with manifest delirium. In the latest Cochrane Review, 4 Randomised Controlled Trials (RCTs) were included to evaluate the treatment effect of typical antipsychotics [43]. Of these only one study (Girard et al. 2018) was in fact an actual treatment study [44•]. Another challenge in the search for effective delirium treatments in existing literature is that many studies evaluating, e.g. antipsychotics accept use of open-label antipsychotics as an escape intervention. Exposure of the placebo group with the open-label use of the study drug (e.g. antipsychotics) will bias the treatment effect towards null. Underlining these challenges, another recent review summarising the literature on haloperidol for the treatment of delirium, found that the evidence for the use of haloperidol to treat delirium in critically ill patients is sparse, of low quality and inconclusive. The review concluded that we have no certainty regarding any beneficial, harmful or neutral effects of haloperidol which is the most commonly used agent to treat delirium [45••]. To conclude, no pharmacological intervention used to treat delirium can be considered evidence based since documentation of effect is not 
available in existing literature. We need to randomise more patients in RCTs with low risk of bias to elucidate the role of pharmacological interventions treatment of delirium.

\section{Non-pharmacological Interventions}

Non-pharmacological interventions are defined as interventions not involving any kind of medical treatment [46]. Non-pharmacological interventions are aimed to prevent or reduce delirium and are interventions such as physical therapy (e.g. mobilization), occupational therapy, cognitive rehabilitation (e.g. re-orientation), sleep promotion therapy (e.g. light and sound therapy), and family involvement. The interventions might consist of a single component or several components (multicomponent).

Currently, no single non-pharmacological intervention for prevention or management of delirium in the ICU has been shown to be effective. This is mostly due to low or moderate quality of evidence and heterogeneity of the studies (e.g. small sample size) and therefore no recommendations can be made for one single component [15••, 47-49].

The Practice Guidelines for the Prevention and Management of Pain, Agitation/Sedation, Delirium, Immobility, and Sleep Disruption in Adult Patients in the ICU (PADIS) from 2018 recommends a multicomponent non-pharmacological intervention including strategies focusing on reducing delirium by cognitive rehabilitation, promoting sleep non-pharmacologically, improving wakefulness (reduce sedation), mobilizing early, and improving vision and hearing [15••].

Elements of different interventions have been "gathered" in a bundle referred to as the ABCDEF-bundle (Assess, Prevent, and Manage Pain (A), Both Spontaneous Awakening Trials (SAT) and Spontaneous Breathing Trials (SBT) (B), Choice of analgesia and sedation (C), Delirium: Assess, Prevent, and Manage (D), Early mobility and Exercise (E)) and Family involvement and empowerment (F) [50]. The bundle focuses on prevention, symptom assessment and management rather than disease processes and is applicable to every ICU patient regardless of admission diagnosis or status [51•]. A large cohort study evaluating the relationship between ABCDEF bundle and patient-centered outcomes showed a significant reduction in incidence of delirium [51•].

A recently published systematic review, though, found no evidence for the effectiveness of non-pharmacological interventions on reducing the incidence or duration of delirium in the ICU [52].

Ultimately, despite limited evidence adapting non-pharmacological interventions encompassing the elements of the ABCDEF bundle seems rational to recommend due to the harmless nature of the intervention and the possible benefit. Further studies are however needed to explore the effectiveness of multicomponent non-pharmacological interventions.

\section{Short- and Long-Term Outcomes of Delirium}

Having delirium in the ICU is associated with longer time on mechanical ventilation, increased length of stay in both the ICU and the hospital and an increased risk of being discharged to a high-dependency facility [53]. Delirium is associated with a higher mortality rate, both in and out of hospital. A longer duration of delirium corresponds to a higher risk. This association is strong and persists after adjustment for relevant covariates such as disease severity and age [53].

Long-term impairments after ICU stay have been coined the post-intensive care syndrome (PICS) [54]. This is an entity often seen after intensive care treatment and consists of cognitive, mental and physical impairments [55]. Symptoms can be persistent for years and can also affect family members.

Cognitive impairments are common after critical illness, and patients who experience delirium while critically ill, have worse cognitive function after discharge. This impairment persists at one year and is of the same magnitude as in patients with mild traumatic brain injury $[53,56,57]$. Psychological symptoms are often experienced after critical illness. Even though depressive symptoms are prevalent after critical illness, the association to delirium is still somewhat unclear [58, 59]. There is, however, a high frequency of depressive symptoms after critical illness and a strong association between delirium in hospitalized patients and depression has been demonstrated [60, 61]. Symptoms of anxiety and post-traumatic stress disorder (PTSD) have been linked with delirium, but evidence is not clear [60]. PTSD has been associated with having memories of delusions which is a key component of delirium [62, 63].

Physical function is typically impaired, even a year after discharge when measured by activities of daily living (ADL) [64]. There is a clear dose response with longer duration of delirium leading to worse function. This may be a consequence of the longer time on ventilator seen in patients with delirium.

Few studies have explored the impact of delirium on health-related quality of life (HRQOL) and found no difference between delirious and non-delirious patients [65, 66]. However, taken the abovementioned declines in function into consideration, it seems rational to assume that delirium in the ICU will have an influence on HRQOL. Further studies are needed to explore delirium's impact on HRQOL. 


\section{Core Outcomes}

Although delirium is recognized as deleterious for patients and treatments are few and insufficient, most delirium research is of low quality and large heterogeneity. Many studies on delirium are of small sample size, unblinded and non-randomized. There are, however, an increasing amount of larger high-quality studies, especially regarding pharmacological treatments. To inform practice, the data from these studies will have to be combined in a solid meta-analysis.

Comparing the results in meta-analyses and reviews is problematic as outcomes are very heterogenous and methods are many [67]. In the light of this, a core outcome set is highly needed in delirium research. A core outcome set describes a set of outcomes to be used within an area of research to improve comparability across studies. The outcomes are identified through a process involving clinicians, patients, funders and researchers [68]. Recently, an international collaboration, the Del-COrS initiative, developed a core outcome set for delirium, both in the ICU and for other settings [69]. For example, delirium was measured in more than ten different ways in the included studies. When the core outcome set is published, it will hopefully increase the quality of reporting and ease comparison between treatment effects in order to create evidence-based guidance for the clinician.

\section{Conclusion}

This review has highlighted several areas in which the evidence is weak. Future research should create better understanding of delirium etiology, pathophysiology and phenotypes (e.g. motor subtypes). Larger studies with low risk of bias are needed to identify possible preventable risk factors of delirium and large, pragmatic RCTs with low risk of bias are needed to identify effective nonpharmacological and pharmacological treatments for delirium. Focus on the delirium burden should also be emphasized by increasing research in long-term consequences of delirium. Finally, we suggest a stronger focus on the patient experiences also known as 'experienced delirium'. The perspectives of patients and relatives should be incorporated in the future search for tools that may diminish the delirium burden.

The quantity of delirium research is constantly increasing. With the growing number of studies and a better framework for research leading to stronger evidence, the outcomes for patients suffering from delirium will most definitely improve in the years to come.
Acknowledgements The authors are initiators, sponsor and co-investigators of the Agents Intervening Against Delirium in Intensive Care Unit (AID-ICU) trial, a randomized clinical trial assessing the benefits and harms of haloperidol on treatment of delirium in patients who are critically ill.

\section{Compliance with Ethical Standards}

Conflict of Interest The authors declare no competing interests.

Human and Animal Rights and Informed Consent This article does not contain any studies with human or animal subjects performed by any of the authors.

\section{References}

Papers of particular interest, published recently, have been highlighted as:

- Of importance

$\bullet$ Of major importance

1. Morandi A, Pandharipande PP, Jackson JC, Bellelli G, Trabucchi M, Ely EW. Understanding terminology of delirium and longterm cognitive impairment in critically ill patients. Best Pract Res Clin Anaesthesiol. 2012;26(3):267-76.

2. European Delirium Association, American Delirium Society. The DSM-5 criteria, level of arousal and delirium diagnosis: inclusiveness is safer. BMC Med. 2014;12(1):141.

3. Kotfis K, Marra A, Ely EW. ICU delirium - a diagnostic and therapeutic challenge in the intensive care unit. Anaesthesiol Intensive Ther. 2018;50(2):160-7.

4. Meagher DJ, Trzepacz PT. Motoric subtypes of delirium. Semin Clin Neuropsychiatry. 2000;5(2):75-85.

5. Krewulak KD, Stelfox HT, Leigh JP, Ely EW, Fiest KM. Incidence and Prevalence of Delirium Subtypes in an Adult ICU: A Systematic Review and Meta-Analysis. Crit Care Med. 2018;46(12):2029-35.

6. Bergeron N, Dubois MJ, Dumont M, Dial S, Skrobik Y. Intensive Care Delirium Screening Checklist: evaluation of a new screening tool. Intensive Care Med. 2001;27(5):859-64.

7. Ely EW, Gautam S, Margolin R, Francis J, May L, Speroff T, et al. The impact of delirium in the intensive care unit on hospital length of stay. Intensive Care Med. 2001;27(12):1892-900.

8. Gélinas C, Bérubé M, Chevrier A, Pun BT, Ely EW, Skrobik Y, et al. Delirium Assessment Tools for Use in Critically Ill Adults: A Psychometric Analysis and Systematic Review. Crit Care Nurse. 2018;38(1):38-49.

9. Andrews L, Silva SG, Kaplan S, Zimbro K. Delirium monitoring and patient outcomes in a general intensive care unit. Am J Crit Care Off Publ Am Assoc Crit-Care Nurses. 2015;24(1):48-56.

10. van den Boogaard M, Pickkers P, van der Hoeven H, Roodbol G, van Achterberg T, Schoonhoven L. Implementation of a delirium assessment tool in the ICU can influence haloperidol use. Crit Care Lond Engl. 2009;13(4):R131.

11. Barr J, Fraser GL, Puntillo K, Ely EW, Gélinas C, Dasta JF, et al. Clinical practice guidelines for the management of pain, agitation, and delirium in adult patients in the intensive care unit. Crit Care Med. 2013;41(1):263-306.

12. Devlin JW, Fong JJ, Fraser GL, Riker RR. Delirium assessment in the critically ill. Intensive Care Med. 2007;33(6):929-40. 
13. van Eijk MMJ, van Marum RJ, Klijn IAM, de Wit N, Kesecioglu J, Slooter AJC. Comparison of delirium assessment tools in a mixed intensive care unit. Crit Care Med. 2009;37(6):1881-5.

14. Spronk PE, Riekerk B, Hofhuis J, Rommes JH. Occurrence of delirium is severely underestimated in the ICU during daily care. Intensive Care Med. 2009;35(7):1276-80.

15.••Devlin JW, Skrobik Y, Gélinas C, Needham DM, Slooter AJC, Pandharipande PP, et al. Clinical Practice Guidelines for the Prevention and Management of Pain, Agitation/Sedation, Delirium, Immobility, and Sleep Disruption in Adult Patients in the ICU. Crit Care Med. 2018;46(9):e825-73. A very comprehensive review of the literature with conclusive recommandations.

16. Grover S, Kate N. Assessment scales for delirium: A review. World J Psychiatry. 2012;2(4):58-70.

17. Meagher DJ, O'Hanlon D, O'Mahony E, Casey PR, Trzepacz PT. Relationship between symptoms and motoric subtype of delirium. J Neuropsychiatry Clin Neurosci. 2000;12(1):51-6.

18. Gunther ML, Jackson JC, Ely EW. Loss of IQ in the ICU brain injury without the insult. Med Hypotheses. 2007;69(6):1179-82.

19. Hopkins RO, Gale SD, Weaver LK. Brain atrophy and cognitive impairment in survivors of Acute Respiratory Distress Syndrome. Brain Inj. 2006;20(3):263-71.

20 Gunther ML, Morandi A, Ely EW. Pathophysiology of delirium in the intensive care unit. Crit Care Clin. 2008;24(1):45-65, viii.

21. Hopkins RO, Jackson JC. Assessing neurocognitive outcomes after critical illness: are delirium and long-term cognitive impairments related? Curr Opin Crit Care. 2006;12(5):388-94.

22 Maldonado JR. Pathoetiological model of delirium: a comprehensive understanding of the neurobiology of delirium and an evidence-based approach to prevention and treatment. Crit Care Clin. 2008;24(4):789-856, ix.

23. Han L, McCusker J, Cole M, Abrahamowicz M, Primeau F, Elie M. Use of medications with anticholinergic effect predicts clinical severity of delirium symptoms in older medical inpatients. Arch Intern Med. 2001;161(8):1099-105.

24. Brummel NE, Girard TD. Delirium in the critically ill patient. Handb Clin Neurol. 2019;167:357-75.

25. Maclullich AMJ, Ferguson KJ, Miller T, de Rooij SEJA, Cunningham C. Unravelling the pathophysiology of delirium: a focus on the role of aberrant stress responses. J Psychosom Res. 2008;65(3):229-38.

26. Dantzer R. Cytokine-induced sickness behaviour: a neuroimmune response to activation of innate immunity. Eur J Pharmacol. 2004;500(1-3):399-411.

27. Perry VH, Cunningham C, Holmes C. Systemic infections and inflammation affect chronic neurodegeneration. Nat Rev Immunol. 2007;7(2):161-7.

28. Herman JP, Figueiredo H, Mueller NK, Ulrich-Lai Y, Ostrander MM, Choi DC, et al. Central mechanisms of stress integration: hierarchical circuitry controlling hypothalamo-pituitary-adrenocortical responsiveness. Front Neuroendocrinol. 2003;24(3):151-80.

29. Otte C, Hart S, Neylan TC, Marmar CR, Yaffe K, Mohr DC. A meta-analysis of cortisol response to challenge in human aging: importance of gender. Psychoneuroendocrinology. 2005;30(1):80-91.

30. Pandharipande P, Shintani A, Peterson J, Pun BT, Wilkinson GR, Dittus RS, et al. Lorazepam is an independent risk factor for transitioning to delirium in intensive care unit patients. Anesthesiology. 2006;104(1):21-6.

31. Pandharipande P, Cotton BA, Shintani A, Thompson J, Pun BT, Morris JA, et al. Prevalence and risk factors for development of delirium in surgical and trauma intensive care unit patients. J Trauma. 2008;65(1):34-41.

32. Fan H, Ji M, Huang J, Yue P, Yang X, Wang C, et al. Development and validation of a dynamic delirium prediction rule in patients admitted to the Intensive Care Units (DYNAMICICU): A prospective cohort study. Int J Nurs Stud. 2019;93:64-73.

33. Green C, Bonavia W, Toh C, Tiruvoipati R. Prediction of ICU Delirium: Validation of Current Delirium Predictive Models in Routine Clinical Practice*. Crit Care Med. 2019;47(3):428-35.

34. Newman MW, O'Dwyer LC, Rosenthal L. Predicting delirium: a review of risk-stratification models. Gen Hosp Psychiatry. 2015;37(5):408-13.

35. Halladay CW, Sillner AY, Rudolph JL. Performance of Electronic Prediction Rules for Prevalent Delirium at Hospital Admission. JAMA Netw Open. 2018;1(4):e181405.

36. Chen J, Yu J, Zhang A. Delirium risk prediction models for intensive care unit patients: A systematic review. Intensive Crit Care Nurs. 2020;60:102880.

37. van den Boogaard M, Pickkers P, Slooter AJC, Kuiper MA, Spronk PE, van der Voort PHJ, et al. Development and validation of PRE-DELIRIC (PREdiction of DELIRium in ICu patients) delirium prediction model for intensive care patients: observational multicentre study. BMJ. 2012;344:e420.

38 van den Boogaard M, Pickkers P. Untangling ICU delirium: is establishing its prevention in high-risk patients the final frontier? Reply to van der Jagt et al. Intensive Care Med. 2014;40(8):1183.

39. Wassenaar A, Schoonhoven L, Devlin JW, van Haren FMP, Slooter AJC, Jorens PG, et al. Delirium prediction in the intensive care unit: comparison of two delirium prediction models. Crit Care. 2018;22(1):1-9.

40. Lonergan E, Britton AM, Luxenberg J, Wyller T. Antipsychotics for delirium. Cochrane Database Syst Rev. 2007;(2):CD005594.

41 Serafim RB, Bozza FA, Soares M, do Brasil PEAA, Tura BR, Ely EW, et al. Pharmacologic prevention and treatment of delirium in intensive care patients: A systematic review. J Crit Care. 2015;30(4):799-807.

42. Collet MO, Caballero J, Sonneville R, Bozza FA, Nydahl P, Schandl A, et al. Prevalence and risk factors related to haloperidol use for delirium in adult intensive care patients: the multinational AID-ICU inception cohort study. Intensive Care Med. 2018;44(7):1081-9.

43. Burry L, Hutton B, Williamson DR, Mehta S, Adhikari NK, Cheng W, et al. Pharmacological interventions for the treatment of delirium in critically ill adults. Cochrane Database Syst Rev. 2019;9:CD011749.

44. Girard TD, Exline MC, Carson SS, Hough CL, Rock P, Gong $\mathrm{MN}$, et al. Haloperidol and Ziprasidone for Treatment of Delirium in Critical Illness. N Engl J Med. 2018;379(26):250616. The most recent RCT of the most abundant phamacological treatment of delirium.

45.•• Barbateskovic M, Krauss SR, Collet MO, Andersen-Ranberg $\mathrm{NC}$, Mathiesen O, Jakobsen JC, et al. Haloperidol for the treatment of delirium in critically ill patients: A systematic review with meta-analysis and Trial Sequential Analysis. Acta Anaesthesiol Scand. 2020;64(2):254-66. A comprehensive review of the evidence for treatment of delirium with haloperidol.

46. Hoffmann TC, Erueti C, Glasziou PP. Poor description of nonpharmacological interventions: analysis of consecutive sample of randomised trials. BMJ [Internet]. 2013 [cited 2020 Nov 16];347. Available from: http://www.bmj.com/content/347/bmj. f3755.

47. Bannon L, McGaughey J, Clarke M, McAuley DF, Blackwood B. Impact of non-pharmacological interventions on prevention and treatment of delirium in critically ill patients: protocol for a systematic review of quantitative and qualitative research. Syst Rev. 2016;5(1):1-9.

48. Wilson JE, Mart MF, Cunningham C, Shehabi Y, Girard TD, MacLullich AMJ, et al. Delirium. Nat Rev Dis Primer. 2020;6(1):1-26. 
49. Rosa RG, Falavigna M, da Silva DB, Sganzerla D, Santos MMS, Kochhann R, et al. Effect of Flexible Family Visitation on Delirium Among Patients in the Intensive Care Unit: The ICU Visits Randomized Clinical Trial. JAMA. 2019;322(3):216-28.

50. Marra A, Ely EW, Pandharipande PP, Patel MB. The ABCDEF Bundle in Critical Care. Crit Care Clin. 2017;33(2):225-43.

51.• Pun BT, Balas MC, Barnes-Daly MA, Thompson JL, Aldrich JM, Barr J, et al. Caring for Critically Ill Patients with the ABCDEF Bundle: Results of the ICU Liberation Collaborative in Over 15,000 Adults. Crit Care Med. 2019;47(1):3-14. A large cohorte study of non-pharmacological prevention and intervention against delirium.

52. Bannon L, McGaughey J, Verghis R, Clarke M, McAuley DF, Blackwood B. The effectiveness of non-pharmacological interventions in reducing the incidence and duration of delirium in critically ill patients: a systematic review and meta-analysis. Intensive Care Med. 2019;45(1):1-12.

53. Ely EW, Shintani A, Truman B, Speroff T, Gordon SM, Harrell $\mathrm{FE}$, et al. Delirium as a predictor of mortality in mechanically ventilated patients in the intensive care unit. JAMA J Am Med Assoc. 2004;291(14):1753-62.

54. Myers EA, Smith DA, Allen SR, Kaplan LJ. Post-ICU syndrome. J Am Acad Physician Assist. 2016;29(4):34-7.

55. Mikkelsen ME, Still M, Anderson BJ, Bienvenu OJ, Brodsky MB, Brummel N, et al. Society of critical care medicine's international consensus conference on prediction and identification of long-term impairments after critical illness. Vol. 48, Critical Care Medicine. Lippincott Williams and Wilkins; 2020. p. 1670-9.

56. Girard TD, Jackson JC, Pandharipande PP, Pun BT, Thompson JL, Shintani AK, et al. Delirium as a predictor of long-term cognitive impairment in survivors of critical illness. Crit Care Med. 2010;38(7):1513-20.

57. Pandharipande PP, Girard TD, Jackson JC, Morandi A, Thompson JL, Pun BT, et al. Long-term cognitive impairment after critical illness. N Engl J Med. 2013;369(14):1306-16.

58. Wolters AE, Peelen LM, Welling MC, Kok L, De Lange DW, Cremer OL, et al. Long-Term Mental Health Problems after Delirium in the ICU. Crit Care Med. 2016;44(10):1808-13.

59. Nouwen MJ, Klijn FAM, van den Broek BTA, Slooter AJC. Emotional consequences of intensive care unit delirium and delusional memories after intensive care unit admission: A systematic review. J Crit Care. 2012;27(2):199-211.
60. Langan C, Sarode DP, Russ TC, Shenkin SD, Carson A, Maclullich AMJ. Psychiatric symptomatology after delirium: a systematic review. Vol. 17, Psychogeriatrics. Blackwell Publishing Ltd; 2017. p. 327-35.

61. Jackson JC, Pandharipande PP, Girard TD, Brummel NE, Thompson JL, Hughes CG, et al. Depression, post-traumatic stress disorder, and functional disability in survivors of critical illness in the BRAIN-ICU study: A longitudinal cohort study. Lancet Respir Med. 2014;2(5):369-79.

62. Svenningsen H, Egerod I, Christensen D, Tønnesen EK, Frydenberg M, Videbech P. Symptoms of posttraumatic stress after intensive care delirium. BioMed Res Int. 2015;2015.

63. Marra A, Pandharipande PP, Patel MB. Intensive Care Unit Delirium and Intensive Care Unit-Related Posttraumatic Stress Disorder. Vol. 97, Surgical Clinics of North America. W.B. Saunders; 2017. p. 1215-35.

64. Brummel NE, Jackson JC, Pandharipande PP, Thompson JL, Shintani AK, Dittus RS, et al. Delirium in the ICU and subsequent long-term disability among survivors of mechanical ventilation. Crit Care Med. 2014;42(2):369-77.

65. van den Boogaard M, Schoonhoven L, Evers AWM, van der Hoeven JG, van Achterberg T, Pickkers P. Delirium in critically ill patients: impact on long-term health-related quality of life and cognitive functioning. Crit Care Med. 2012;40(1):112-8.

66. Wolters AE, van Dijk D, Pasma W, Cremer OL, Looije MF, de Lange DW, et al. Long-term outcome of delirium during intensive care unit stay in survivors of critical illness: a prospective cohort study. Crit Care Lond Engl. 2014;18(3):R125.

67. Clarke M, Williamson PR. Core outcome sets and systematic reviews. Syst Rev. 2016;5(1):11.

68. Williamson PR, Altman DG, Blazeby JM, Clarke M, Devane D, Gargon E, et al. Developing core outcome sets for clinical trials: Issues to consider. Trials. 2012;13(1):132.

69. Rose L, Agar M, Burry L, Campbell N, Clarke M, Lee J, et al. A core outcome set for research evaluating interventions to prevent and/or treat delirium in critically Ill adults: an international consensus study (Del-COrS). Crit Care Med. 2021;49(9):1535-46.

Publisher's Note Springer Nature remains neutral with regard to jurisdictional claims in published maps and institutional affiliations. 